\title{
Mycoplasmal infection in a guigna (Leopardus guigna) from central Chile
}

\author{
Diana Echeverry ${ }^{\mathrm{a}, \mathrm{b}, \mathrm{c}}$, Sebastián Llanos-Soto ${ }^{\mathrm{b}, \mathrm{c}}$, Cristina Palma $^{\mathrm{d}}$, Linda Castillo $^{\mathrm{d}}$, Tomás Casanova ${ }^{\mathrm{d}}$, \\ Ignacio Troncoso ${ }^{\mathrm{c}}$, Manuel Valdés ${ }^{\mathrm{c}}$, Fidel O. Castro ${ }^{\mathrm{a}^{*}}$, Daniel González-Acuña ${ }^{\mathrm{c}}$
}

\begin{abstract}
Routine blood analysis indicated the presence of Mycoplasma-like bodies in a guigna (Leopardus guigna). Evidence of infection with Candidatus Mycoplasma haemominutum was found in blood samples using PCR and DNA sequencing of the 16S rRNA gene of Mycoplasma. Mycoplasma spp. are documented in cats but their role in the transmission of Mycoplasma to guigna populations requires investigation.

Key words: bacteria, domestic animals, Felidae, vulnerable.
\end{abstract}

\section{INTRODUCTION}

Urbanization and natural environments is an opportunity for the transmission of infectious diseases to native wildlife (Valenzuela-Sanchez \& Medina-Vogel 2014). The illegal possession of wildlife and the free-roaming of domestic species in rural areas is well known to have a substantial impact on several aspects of wild animal ecology including habitat use, activity patterns, and hostpathogen interactions (Hwang et al 2018). Currently, information about pathogens in populations of Chilean wildlife is limited and further research is necessary to properly understand the consequences of these infections (Llanos-Soto \& González-Acuña 2019).

Haemotropic mycoplasmas (haemoplasmas) are small epierythrocytic bacteria that infect a wide variety of mammalian species, including domestic cats (Sykes 2010). Four haemoplasmas species are identified to infect domestic cats: Mycoplasma haemofelis (Mhm), Candidatus Mycoplasma the associated introduction of domestic animals in haemominutum $(\mathrm{CMhm})$, Candidatus Mycoplasma turicensis (CMt) and Candidatus Mycoplasma haematoparvum-like (Sykes 2010). In Chile, mycoplasmal infection is common among domestic animals, with findings from a report by Walker et al (2016) indicating a prevalence of $15.1 \%$ in cats living in the southern region of the country. Nonetheless, the detection in wild species such as Darwin's Fox (Lycalopex fulvipes) and guigna

Received: 07.08.2020.

Accepted: 26.01.2021.

aLaboratorio de Biotecnología, Departamento de Ciencia Animal. Facultad de Ciencias Veterinarias, Universidad de Concepción, Chillán, Chile.

bLaboratorio de Vida Silvestre, Departamento de Ciencia Animal. Facultad de Ciencias Veterinarias, Universidad de Concepción, Chillán, Chile.

'Laboratorio de Enfermedades y Parásitos de Fauna Silvestre, Departamento de Ciencia Animal. Facultad de Ciencias Veterinarias, Universidad de Concepción, Chillán, Chile.

dLaboratorio de Patología Clínica Animal, Departamento de Ciencias Clínicas, Facultad de Ciencias Veterinarias, Universidad de Concepción, Chillán, Chile.

*Corresponding author: FO Castro; Av. Vicente Méndez 595, Chillán, Chile; fidcastro@udec.cl
(Leopardus guigna), is rather recent (Cabello et al 2013, Walker et al 2016, Di Cataldo et al 2020). The guigna inhabits the temperate rainforests of central and southern Chile and it is currently categorised as Vulnerable by the IUCN (International Union for Conservation of Nature) (Gálvez et al 2013). Here, we reported the evidence of infection with Candidatus Mycoplasma haemominutum $(\mathrm{CMhm})$ in a guigna illegally kept as a pet.

\section{MATERIAL AND METHODS}

In August 21, 2018, a male adult guigna (Leopardus guigna) was confiscated by the Livestock and Agriculture Service (SAG) and brought in to the Wildlife Rehabilitation Centre, Universidad de Concepción, Chillán. The animal was being illegally kept in a warehouse by a local family from El Carmen ( $\left.36^{\circ} 53^{\prime} \mathrm{S}, 72^{\circ} 01^{\prime} \mathrm{W}\right)$. On arrival, the individual did not exhibit any behavioural or physical anomalies and was unafraid of human handling. An anaesthesia protocol with $20 \mathrm{mg} / \mathrm{kg}$ of demedetomidine and $0.3 \mathrm{mg} / \mathrm{kg}$ of methadone IM was performed to collect blood from the saphenous vein for biochemical and haematological testing, as it is routinely carried out for all animals entering quarantine on their arrival to the rehabilitation centre (Tayari et al 2015). Blood smears were also prepared and stained with Giemsa stain for microscopic observation.

DNA extraction from blood was carried out using the DNAeasy Blood \& Tissue kit (Qiagen) and according to the manufacturer's instructions. DNA templates obtained through this protocol were amplified in a thermocycler (MultiGene ${ }^{\mathrm{TM}}$ OptiMax Thermal Cycler, Labnet) and the 16S rRNA and RNAseP gene of Mycoplasma was targeted using primers described in table 1 . The DNA sample was also analysed to detect the presence of other pathogens (table 1). The amplificated PCR products were run in 1\% agarose gel, purified using the SigmaSpin TM Post-Reaction Purification Columns (Sigma-Aldrich), and analysed on the ABI Prism 310 Genetic Analyzer (Applied Biosystems). The sequence corresponding to the $16 \mathrm{~S}$ rRNA was aligned to a single consensus sequence by ProSeq 3.5 software and subject to comparison with the GenBank database of National Center for Biotechnology Information (NCBI). 
Table 1. Primer pairs used for amplification of pathogens of DNA sample from guigna (Leopardus guigna).

\begin{tabular}{|c|c|c|c|c|}
\hline Primer & Sequence & $\begin{array}{l}\text { Size } \\
(\mathrm{pb})\end{array}$ & Organims & Reference \\
\hline $\begin{array}{l}g l t A \\
\text { CS78F } \\
\text { CS323R }\end{array}$ & $\begin{array}{c}\text { 5'-GCAAGTATCGGTGAGGATGTAAT-3' } \\
\text { 5'-GCTTCCTTAAAATTCAATAAATCAGGAT-3' }\end{array}$ & 401 & Rickettsia & Labruna et al 2004 \\
\hline $\begin{array}{l}\text { EHR12SD } \\
\text { EHR16SR }\end{array}$ & $\begin{array}{l}\text { 5'-GGTACCYACAGAAGAAGTCC-3' } \\
\text { 5'-TAGACTCATCGTTTA-3' }\end{array}$ & 345 & Anaplasmataceae & Parola et al 2000 \\
\hline $\begin{array}{l}\text { HBT-F } \\
\text { HBT-R }\end{array}$ & $\begin{array}{l}\text { 5'-ATACGGCCCATATTCCTACG-3' } \\
\text { 5'-TGCTCCACCACTTGTTCA-3' }\end{array}$ & 618 & $\begin{array}{l}\text { Candidatus Mycoplasma } \\
\text { haemominutum }\end{array}$ & $\begin{array}{l}\text { Criado-Fornelio } \\
\quad \text { et al } 2003\end{array}$ \\
\hline $\begin{array}{l}\text { RNAsePF } \\
\text { RNAsePR }\end{array}$ & $\begin{array}{l}\text { 5'-CTGCGATGGTCGTAATGTTG-3' } \\
\text { 5'-GAGGAGTTTACCGCGTTTCA-3' }\end{array}$ & 175 & $\begin{array}{l}\text { Candidatus Mycoplasma } \\
\text { haemominutum }\end{array}$ & Tasker et al 2003 \\
\hline $\begin{array}{l}\text { RNAseP-Cmh F } \\
\text { RNAseP-Cmh R }\end{array}$ & $\begin{array}{l}\text { 5'-CTCTCGTCATTTCTGCAGAACGTC-3' } \\
\text { 5'-CGCTTGCACAGTCTGAGATGA-3' }\end{array}$ & 175 & $\begin{array}{l}\text { Candidatus Mycoplasma } \\
\text { haemominutum }\end{array}$ & This study \\
\hline
\end{tabular}

Mycoplasma sequences reported in domestic and wild felines in the GenBank were used for phylogenetic analysis. Twenty sequences were used to perform the alignment together with the consensus sequence using the ClustalW algorithm (Thompson et al 1994) and phylogenetic trees were constructed based on neighbour-joining, maximum likelihood and Bayesian methods using a GTR + I + G model. Maximum-likelihood analysis was conducted using MEGA 7.0 (Kumar et al 2016) and Bayesian inference analysis was performed with Mr. Bayes 3.1.253 (Ronquist et al 2012). The data set was resampled 1,000 times to generate bootstrap values. Software FigTree 1.4.4 was used for visualisation.

\section{RESULTS AND DISCUSSION}

Results from the blood analysis showed no haematological or biochemical alterations, however, blood smears revealed the presence of Mycoplasma-like bodies in erythrocytes (figure 1). The sequence obtained from the guigna in this study belongs to Candidatus Mycoplasma haemominutum (CMhm) (Accession number: MT772012) (figure 2). Primers reported and designed for the Mycoplasma sp RNAseP gene did not amplify any sequences. To our knowledge, there are no reports on the amplification of the RNAseP gene for CMhm. During the analysis, sequences were clustered in different groups, namely group A (CMhm), group B (Mhf), group C (Candidatus Mycoplasma turicensis - CMt) and group D (Mycoplasma sp).

The sequence obtained in this study was positioned within group A, which is also shared with haemoplasma sequences found in Felis silvestris catus from Chile. The context in which the guigna was found could have resulted in increased exposure to pathogens from cats, including Mycoplasma. Currently, there are few studies accurately addressing Mycoplasma transmission in wild animals in Chile. However, the mechanism of transmission of haemoplasmas among wild and domestic cats has not been

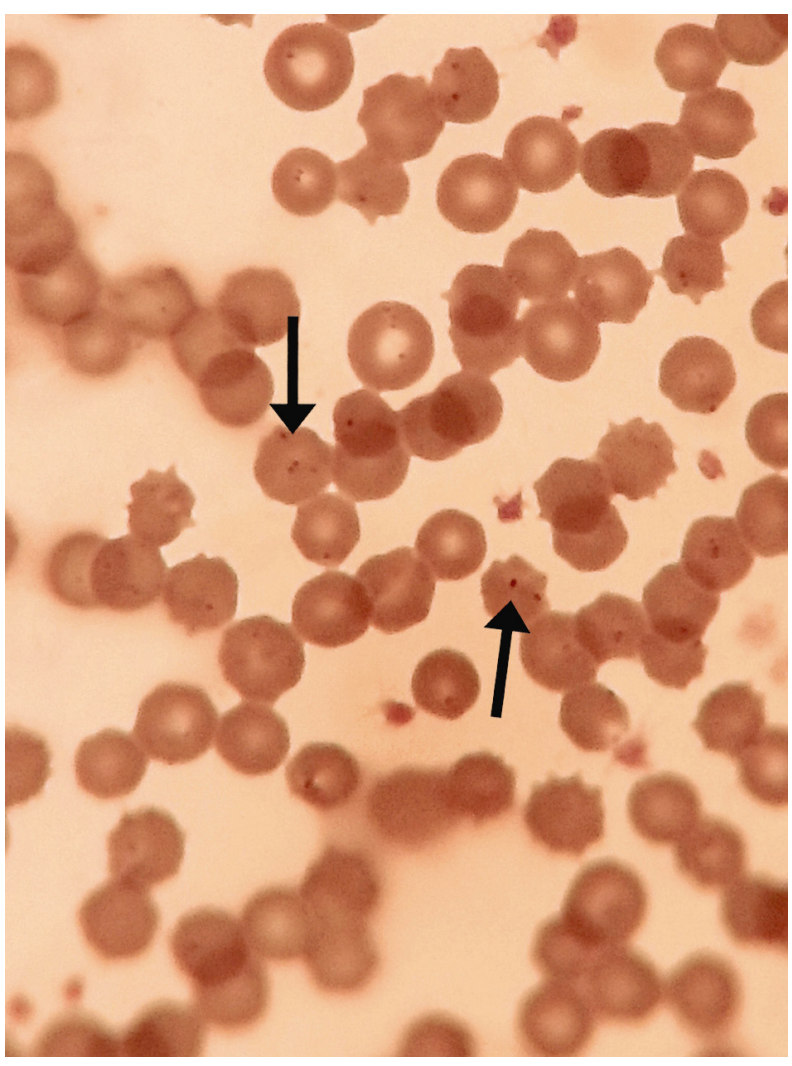

Figure 1. Mycoplasma-like bodies in blood of guigna (Leopardus guigna).

elucidated, a recent study suggests that their interaction might be not linked to exposure to haemoplasmatransmitting vectors (Sacristan et al 2019). Mycoplasma infections can cause disease in domestic cats but just in rare cases there have been reports documenting clinical signs associated with this pathogen in wild carnivores (Criado-Fornelio et al 2003). In this report, the guigna showed no clinical signs which is also the case for most studies in carnivores. However, the animal died two weeks 


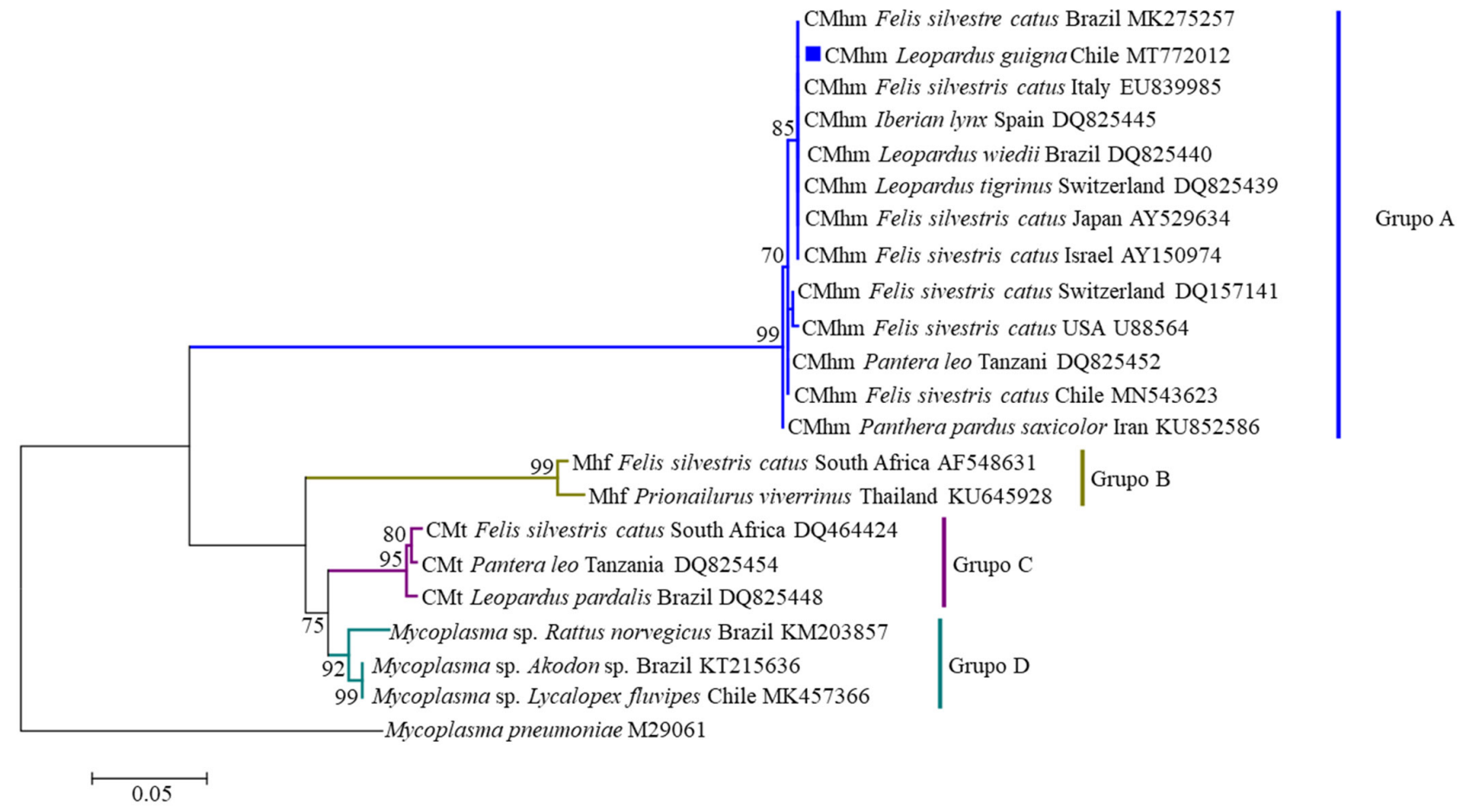

Figure 2. Maximum likelihood tree of 505 bp of the 16S rRNA Mycoplasma gene for guigna. M. pneumonia sequence was used as outgroup. The data set was resampled 1000 times to generate bootstrap percentage values and bootstrap values of $\geq 70$ are printed at the nodes of the tree. $(\square)$ Blue square mark guigna ntST from the present study (Genbank access number MT772012). The Bayesian phylogenetic tree was congruent. The four phylogenetic (taxonomic) groups are labelled (A-D).

later of unknown causes. Mycoplasma infection was not cause of death according to necropsy. Some studies have indicated that domestic cats infected with retroviruses are more susceptible to acquire haemoplasmas and likely to develop more severe clinical signs (Luria et al 2004), which is concerning considering that retroviral infection is already documented in guignas in Chile (Mora et al 2015). Nonetheless, there is no information about guignas co-infected with haemoplasmas and retroviruses. Further investigations are needed to evaluate the health status of guigna populations along its distribution and determine whether infection with Mycoplasma spp. could pose a threat to guigna conservation.

\section{REFERENCES}

Cabello J, Altet L, Napolitano C, Sastre N, Hidalgo E, et al. 2013. Survey of infectious agents in the endangered Darwin's fox (Lycalopex fulvipes): High prevalence and diversity of hemotrophic mycoplasmas. Vet Microbiol 167, 448-454.

Criado-Fornelio A, Martinez-Marcos A, Buling-Sarana A, Barba-Carretero JC. 2003. Presence of Mycoplasma haemofelis, Mycoplasma haemominutum and piroplasmids in cats from southern Europe: a molecular study. Vet Microbiol 93, 307-317.

Di Cataldo S, Hidalgo-Hermoso E, Sacristán I, Cevidanes A, Napolitano C, et al. 2020. Hemoplasmas Are Endemic and Cause Asymptomatic Infection in the Endangered Darwin's Fox (Lycalopex fulvipes). Appl Environ Microbiol 86, 1-13.
Gálvez N, Hernández F, Laker J, Gilabert H, Petitpas, et al. 2013. Forest cover outside protected areas plays an important role in the conservation of the vulnerable guiña Leopardus guigna. Oryx 47, 251-258.

Hwang J, Gottdenker NL, Oh D, Nam H, Lee H, et al. 2018. Disentangling the link between supplemental feeding, population density, and the prevalence of pathogens in urban stray cats. Peer J 6:e4988.

Kumar S, Stecher G, Tamura K. 2016. MEGA7: molecular evolutionary genetics analysis version 7.0 for bigger datasets. Mol Biol Evol 33, 1870-1874.

Labruna MB, Whitworth T, Horta MC, Bouyer DH, McBride JW, et al. 2004. Rickettsia species infecting Amblyomma cooperi ticks from an area in the state of Sao Paulo, Brazil, where Brazilian spotted fever is endemic. J Clin Microbiol 42, 90-98.

Llanos-Soto S, González-Acuña D. 2019. Knowledge about bacterial and viral pathogens present in wild mammals in Chile: a systematic review. Rev Chilena Infectol 36, 195-218.

Luria BJ, Levy JK, Lappin MR, Breitschwerdt EB, Legendre AM, et al. 2004. Prevalence of infectious diseases in feral cats in Northern Florida. J Feline Med Surg 6, 287-296.

Mora M, Napolitano C, Ortega R, Poulin E, Pizarro-Lucero J. 2015. Feline immunodeficiency virus and feline leukemia virus infection in free-ranging guignas (Leopardus guigna) and sympatric domestic cats in human perturbed landscapes on Chiloé Island, Chile. J Wildl Dis 51, 199-208.

Parola P, Roux V, Camicas JL, Baradji I, Brouqui P, et al. 2000. Detection of Ehrlichiae in African ticks by polymerase chain reaction. Trans R Soc Trop Med Hyg 94, 707-708.

Ronquist F, Teslenko M, Van Der Mark P, Ayres DL, Darling A, et al. 2012. MrBayes 3.2: efficient bayesian phylogenetic inference and model choice across a large model space. Syst Biol 61, 539-542. 
Sacristán I, Acuña F, Aguilar E, García S, López MJ, et al. 2019. Assessing cross-species transmission of hemoplasmas at the wild-domestic felid interface in Chile using genetic and landscape variables analysis. Sci Rep 9, 16816

Sykes JE. 2010. Feline hemotropic mycoplasmas. J Vet Emerg Crit Care 20, 62-69.

Tasker S, Helps CR, Day MJ, Harbour DA, Shaw SE, et al. 2003. Phylogenetic analysis of hemoplasma species: an international study. J Clin Microbiol 41, 3877-3880.

Tayari H, Vannozzi I, Breghi G, Briganti A. 2015. Methadone and dexmedetomidine combination as premedicant agents for ovariectomy in cats. Am J Anim Vet Sci 10, 101
Thompson JD, Higgins DG, Gibson TJ. 1994. CLUSTAL W: improving the sensitivity of progressive multiple sequence alignment through sequence weighting, position-specific gap penalties and weight matrix choice. Nucleic Acids Res 22, 4673-4680.

Valenzuela-Sánchez A, Medina-Vogel G. 2014. Importancia de las enfermedades infecciosas para la conservación de la fauna silvestre amenazada de Chile. Gayana (Concepción) 78, 57-69.

Walker R, Morera F, Gómez M, Pereira NR, Martínez P, et al. 2016. Prevalence, risk factor analysis, and hematological findings of hemoplasma infection in domestic cats from Valdivia, Southern Chile. Comp Immunol Microbiol Infect Dis 46, 20-26. 